\title{
$m a g A$ is not a specific virulence gene for Klebsiella pneumoniae strains causing liver abscess but is part of the capsular polysaccharide gene cluster of $K$. pneumoniae serotype $\mathrm{K} 1$
}

Klebsiella pneumoniae is an important emerging pathogen in community-acquired liver abscesses worldwide, especially in Taiwan, Asia and the USA (Chang \& Chou, 1995; Okano et al., 2002; Lim et al., 2003; Lederman \& Crum, 2005). The prevalence of $K$. pneumoniae in cases of pyogenic liver abscess is as high as $78 \%$ in Taiwan and $41 \%$ in the USA (Tsay et al., 2002; Rahimian et al., 2004). Our group has observed that among our K. pneumoniae liver abscess cases, 85 of $134(63 \cdot 4 \%)$ were caused by serotype K1 K. pneumoniae and 12 of the $14(85 \cdot 7 \%)$ cases with complications of endophthalmitis were serotype $\mathrm{K} 1$, including $\mathrm{K} 1$ isolates which are highly resistant to neutrophil phagocytosis (Fung et al., 2002; Lin et al., 2004).

Further investigation by Fang et al. (2004) has identified a novel virulence gene, magA, that causes $K$. pneumoniae liver abscess and septic metastatic complications. Among the isolates in Fang's study, 52 of 53 (98\%) liver abscess isolates carried this specific virulence gene and the presence of one magA-negative isolate was suggested to be due to the patient's underlying disease of liver cirrhosis and hepatic failure. Thus Fang et al. (2004) concluded that $m a g A$ is an essential virulence gene for $K$. pneumoniae strains causing liver abscess and could be used as a diagnostic tool. Unfortunately, the authors did not discuss the correlation of virulence between the novel gene and serotype specificity.

In a recent article in the Journal of Medical Microbiology, Struve et al. (2005) tried to investigate the above correlation and found that, of the 495 Klebsiella isolates from a worldwide collection isolated from unknown different sites, all 39 magA-positive isolates were of serotype $\mathrm{K} 1$ and none of the 456 non-K1 serotypes contained magA. They concluded that
magA is restricted to the capsular gene cluster of serotype K1. Since all the 495 isolates were non-liver abscess isolates, the presence of $m a g A$ in non-K1 liver abscess isolates has not been studied. One may argue that all liver abscess isolates may have the magA virulence gene. Our group has sequenced the whole $\mathrm{K} 1$ capsular gene cluster (GenBank accession no. AY762939; Fig. 1). Thus we have tried to investigate the prevalence of magA among serotypes $\mathrm{K} 1, \mathrm{~K} 2$ and other serotypes from liver abscess patients. Our results further confirmed those of Struve et al. (2005) indicating that magA is only present in serotype K1 liver and non-liver abscess isolates.

In the present study, we investigated the incidence of magA among $134 \mathrm{~K}$. pneumoniae liver abscess isolates (Fung et al., 2002) and 4 strains of K. pneumoniae from the ATCC. Nested PCRs for magA detection were designed according to Fang et al. (2004). The sequence of the two primer sets were as follows: outer forward 5'-GGTGCTCTTTACATCATTGC-3' and outer reverse $5^{\prime}$-GCAATGGCCATTTGCGTAAG-3'; and inner forward 5'-CGCCGCAAATACGAGAAGTG-3' and inner reverse 5' -GCAATCGAAGTGAAGAGTGC. Control strains of serotype K1 (ATCC 4208), K2 (ATCC 9997), K3 (ATCC 13883) and K6 (ATCC 700603) were included for PCR. Results obtained from PCR amplification showed that only K1 isolates were positive for magA (Table 1). All other capsular serotypes were negative for magA. Thus we strongly agree with Struve et al. (2005) that the K1 capsule rather than magA is the important virulence factor for $K$. pneumoniae strains causing liver abscess and that magA is a part of the gene cluster involved in K1 capsule formation. According to studies done on our collection of K. pneumoniae liver abscess isolates, more than $36 \%$ of the cases would have been missed if magA was used as a diagnostic tool. Although $86 \%$ of the cases with complications were caused by serotype $\mathrm{K} 1$ and tested positive for $m a g A$, only 14 cases were collected in our previous study (Fung et al., 2002). The usefulness of magA for the detection of liver abscess and septic metastatic complications needs to be studied further.

We conclude that magA is a component of $\mathrm{K} 1$ capsule formation, but is not an independent virulence gene in $K$. pneumoniae strains causing liver abscess. The $\mathrm{K} 1$ capsule rather than magA is an important virulence factor.

Table 1. PCR amplification of $m a g A$ among different $\mathrm{K}$ serotypes of $K$. pneumoniae isolated from liver abscess patients

\begin{tabular}{|lrc|}
\hline $\begin{array}{l}\text { Serotype } \\
(\text { K })\end{array}$ & $\begin{array}{r}\text { No. of } \\
\text { isolates }\end{array}$ & $\begin{array}{c}\text { Isolates } \\
\text { positive for } \\
\text { magA (\%) }\end{array}$ \\
\hline K1 & 86 & $86(100)$ \\
K2 & 20 & 0 \\
K16 & 6 & 0 \\
K21 & 4 & 0 \\
K28 & 4 & 0 \\
K54 & 3 & 0 \\
K5 & 2 & 0 \\
K29 & 2 & 0 \\
K38 & 2 & 0 \\
K39 & 2 & 0 \\
K57 & 2 & 0 \\
K68 & 1 & 0 \\
K3 & 1 & 0 \\
K6 & 1 & 0 \\
NT & 2 & 0 \\
Total & 138 & $86(63 \cdot 4) \dagger$ \\
\hline
\end{tabular}

${ }^{*}$ Isolates included control strains of serotype K1 (ATCC 4208), K2 (ATCC 9997), K3 (ATCC 13883) and K6 (ATCC 700603). tPercentage excluded ATCC control strains. 


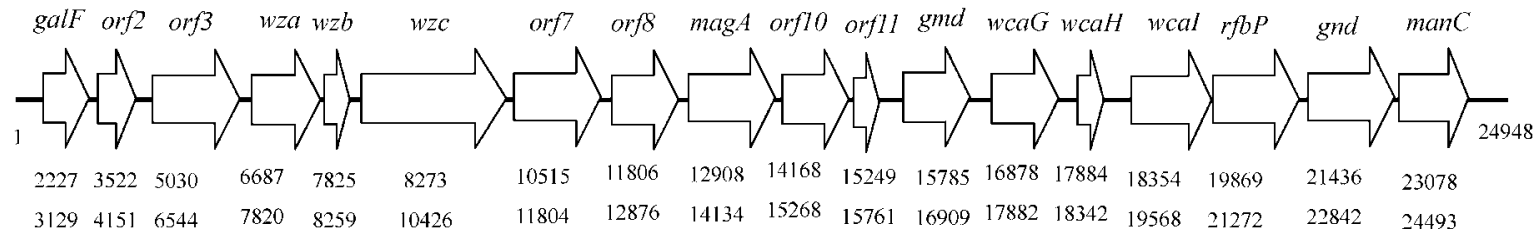

\author{
Gall: UTP-glucose-1-phosphate uridyltransferase \\ ORF2: Hypothetical protein \\ ORF3: Hypothetical protein \\ Wza: Putative capsule polysaccharide export protein \\ Wzb: Protein-tyrosine-phosphatase \\ Wze: Tyrosine-protein kinase \\ ORF7: Hypothetical protein \\ ORF8: Hypothetical protein \\ MagA: Hypothetical protein
}

\begin{abstract}
ORI10: ITypothetical protein
ORF11: Hypothetical protein

Gmd: GDP-D -mannose dehydratase

WcaG: GDPfucose synthetase

WcaH: GDPmannose mannosylhydrolase

Weal: Glycosyltransferase

RfbP: Glycosyltransferase

Gnd: Phosphogluconate dehydrogenase

ManC: Mannose-1-phosphate guanylyltransferase
\end{abstract}

Fig. 1. Gene cluster for K1 capsular polysaccharide (GenBank accession no. AY762939), showing genes with known and unknown functions.

\section{Acknowledgements}

This work is supported by a grant from the National Science Council (NSC94-2320-B-400-002).

\section{Kuo-Ming Yeh, ${ }^{1,2}$ Feng-Yee Chang, ${ }^{1}$ Chang-Phone Fung, ${ }^{3}$ Jung-Chung Lin ${ }^{1}$ and L. K. Siu ${ }^{4}$}

${ }^{1}$ Division of Infectious Diseases and Tropical Medicine, Department of Internal Medicine, Tri-Service General Hospital, Taiwan

${ }^{2}$ Graduate Institute of Medical Sciences, National Defense Medical Center, Taiwan

${ }^{3}$ Section of Infectious Diseases, Department of Medicine, Taipei Veterans General Hospital and National Yang-Ming University, Taiwan

${ }^{4}$ Division of Clinical Research, National Health Research Institutes, NDMC, Min-Chuan East Rd, Neihu, Taipei, Taiwan

Correspondence: L. K. Siu (Iksiu@nhri.org.tw)
Chang, F. Y. \& Chou, M. Y. (1995).

Comparison of pyogenic liver abscesses caused by Klebsiella pneumoniae and non- $K$. pneumoniae pathogens. J Formos Med Assoc 94, 232-237.

Fang, C. T., Chuang, Y. P., Shun, C. T., Chang, S. C. \& Wang, J. T. (2004).A novel virulence gene in Klebsiella pneumoniae strains causing primary liver abscess and septic metastatic complications. J Exp Med 199, 697-705.

Fung, C. P., Chang, F. Y., Lee, S. C., Hu, B. S., Kuo, B. I., Liu, C. Y., Ho, M. \& Siu, L. K. (2002). A global emerging disease of Klebsiella pneumoniae liver abscess: is serotype $\mathrm{K} 1$ an important factor for complicated endophthalmitis? Gut 50, 420-424.

Lederman, E. R. \& Crum, N. F. (2005).

Pyogenic liver abscess with a focus on Klebsiella pneumoniae as a primary pathogen: an emerging disease with unique clinical characteristics. Am J Gastroenterol 100, 322-331.

Lim, S. W., Lee, E. J., Lee, S. W., Kim, S. M., Kim, J. H., Kim, B. J., Jang, B. I., Kim, T. N. \& Chung, M. K. (2003). [Clinical significance of Klebsiella pneumoniae in liver abscess]. Korean J Gastroenterol 42, 226-231.
Lin, J. C., Chang, F. Y., Fung, C. P., Xu, J. Z., Cheng, H. P., Wang, J. J., Huang, L. Y. \& Siu, L. K. (2004). High prevalence of phagocytic-resistant capsular serotypes of Klebsiella pneumoniae in liver abscess. Microbes Infect 6, 1191-1198.

Okano, H., Shiraki, K., Inoue, H. \& 8 other authors (2002). Clinicopathological analysis of liver abscess in Japan. Int J Mol Med $\mathbf{1 0}$, 627-630.

Rahimian, J., Wilson, T., Oram, V. \& Holzman, R. S. (2004). Pyogenic liver abscess: recent trends in etiology and mortality. Clin Infect Dis 39, 1654-1659.

Struve, C., Bojer, M., Nielsen, E. M., Hansen, D. S. \& Krogfelt, K. A. (2005). Investigation of the putative virulence gene magA in a worldwide collection of 495 Klebsiella isolates: $m a g A$ is restricted to the gene cluster of Klebsiella pneumoniae capsule serotype K1. J Med Microbiol 54, 1111-1113.

Tsay, R. W., Siu, L. K., Fung, C. P. \& Chang, F. Y. (2002). Characteristics of bacteremia between community-acquired and nosocomial Klebsiella pneumoniae infection: risk factor for mortality and the impact of capsular serotypes as a herald for community-acquired infection. Arch Intern Med 162, 1021-1027. 